\title{
Anmerkungen zur Schreibweise
}

Die vorliegende Studie basiert auf deutschen, englischen, französischen, türkischen und osmanischen Quellen. Bei Übertragungen von Fremdsprachenzitaten ins Deutsche, denen keine entsprechende Quellenangabe in den Fußnoten folgt, handelt es sich um eigene Übersetzungen. Englische und französische Quellen sind meist in der Originalsprache zitiert, türkische Quellen in Übersetzung. Deutsche Übersetzungen der verwendeten türkischen Literaturtitel finden sich im Literaturverzeichnis, nicht jedoch in den Fußnoten. Bei alten Schreibweisen und kleineren Ungenauigkeiten im Deutschen oder in Fremdsprachenzitaten erfolgt der Lesbarkeit halber keine Kennzeichnung [sic]. Osmanische Begriffe und Zitate werden in einfacher Übertragung in das moderne türkische Schriftsystem wiedergegeben und basieren in den meisten Fällen auf bereits verfügbaren Transkriptionen. Auf die Angabe der Ursprungsfassung in osmanischer Schrift oder eine Transliteration wurde verzichtet, da der Fokus der Analyse auf dem Sinngehalt liegt. Die Schreibweise von türkischen Personennamen und Titeln folgt der heutigen türkischen Gepflogenheit, einschließlich der Sonderzeichen: zum Beispiel Esat Paşa anstelle von Esad Pascha. Da mit Inkrafttreten des Familiennamensgesetzes im Januar 1935 in der Türkei Nachnamen eingeführt und osmanische Titel abgeschafft wurden, sind die Namen der betroffenen Personen bei Erstnennung mit ihrem späteren Nachnamen in Klammern versehen, beispielsweise Halide Edib (Adivar). Bei der ersten Namensnennung thematisch bedeutsamer türkischer Akteure werden zudem in Klammern die Lebensdaten angegeben, soweit bekannt. Sollten sich die Jahreszahlen hinter einem Namen nicht auf die Lebenszeit dieser Person beziehen, sondern etwa auf ihre Regierungszeit, so ist dies durch den fehlenden Asterisk vor der ersten Jahreszahl ersichtlich. Die Verwendung von Ortsbezeichnungen orientiert sich am heutigen türkischen Gebrauch und ist in der deutschen Schreibweise wiedergegeben, also etwa Edirne anstatt Adrianopel, Istanbul anstelle von Konstantinopel, Izmir statt Smyrna. Davon ausgenommen bleiben direkte Quellenzitate. 
